\title{
Nucleon-Nucleon Phase Shifts and Pairing in Neutron Matter and Nuclear Matter
}

\author{
$\varnothing$. Elgarø $\mathrm{y}^{a}$ and M. Hjorth-Jensen ${ }^{b}$ \\ ${ }^{a}$ Department of Physics, University of Oslo, N-0316 Oslo, Norway \\ ${ }^{b}$ Nordita, Blegdamsvej 17, DK-2100 København Ø, Denmark
}

\begin{abstract}
We consider ${ }^{1} S_{0}$ pairing in infinite neutron matter and nuclear matter and show that in the lowest order approximation, where the pairing interaction is taken to be the bare nucleon-nucleon (NN) interaction in the ${ }^{1} S_{0}$ channel, the pairing interaction and the energy gap can be determined directly from the ${ }^{1} S_{0}$ phase shifts. This is due to the almost separable character of the nucleonnucleon interaction in this partial wave. Since the most recent NN interactions are charge-dependent, we have to solve coupled gap equations for proton-proton, neutron-neutron, and neutron-proton pairing in nuclear matter. The results are, however, found to be close to those obtained with charge-independent potentials.
\end{abstract}

PACS number(s): 21.30.-x, 21.65.+f, 26.60.+c

Recently, there has been renewed interest in the pairing problem in neutron matter and neutron-rich nuclei. The superfluid properties of neutron matter is of importance in the study of neutron stars [1], while pairing in neutron-rich systems is of relevance for the study of heavy nuclei close to the drip line [2] and the light halo nuclei [3]. Much effort has gone into calculating the superfluid energy gap in dilute neutron matter [4 [4. Most of these studies, e.g., those of Refs. [4, 6, 8] have been carried out using pairing matrix elements given by the bare nucleonnucleon (NN) interaction. Many of the same authors have calculated the ${ }^{1} S_{0}$ gap in nuclear matter, which has also been the subject of recent relativistic formulations of the pairing problem [9 11].

Even though it is a long time since Clark et al. 12. showed that the effects of density and spin-density fluctuations must be included in the pairing interaction, and there has been much progress in that direction recently [13, 14, we will here focus on the situation at the level of the bare interaction. In this lowest-order approximation to the problem it has been found that results for the ${ }^{1} S_{0}$ energy gap in neutron matter and in nuclear matter are almost independent of the choice of NN interaction. We aim at explaining how this can be understood directly from the measured properties of the free $\mathrm{NN}$ interaction. Moreover, although a relation between the pairing gap and NN phase shifts was obtained almost forty years ago by Emery and Sessler 15 (see also Hoffberg et al. [16]), in this work we wish to focus on the near interaction independence of the results for the energy gap at the Fermi level, and try to explain this from the NN scattering data directly.

The energy gap in infinite matter is obtained by solving the BCS equation for the gap function $\Delta(k)$.

$$
\Delta(k)=-\frac{1}{\pi} \int_{0}^{\infty} d k^{\prime} k^{\prime 2} V\left(k, k^{\prime}\right) \frac{\Delta\left(k^{\prime}\right)}{E\left(k^{\prime}\right)},
$$

where $V\left(k, k^{\prime}\right)$ is the bare momentum-space $\mathrm{NN}$ interaction in the ${ }^{1} S_{0}$ channel, and $E(k)$ is the quasiparticle energy given by $E(k)=\sqrt{\left(\epsilon(k)-\epsilon\left(k_{F}\right)\right)^{2}+\Delta(k)^{2}}$, where $\epsilon(k)$ is the single-particle energy of a neutron with momentum $k$, and $k_{F}$ is the Fermi momentum. Medium effects should be included in $\epsilon(k)$, but we will use free single-particle energies $\epsilon(k)=k^{2} / 2 m$, where $m$ is the neutron rest mass, to avoid unnecessary complications. And in neutron matter, at least at the densities considered here, Brueckner-type calculations [7] indicate that in-medium single-particle energies do not differ much from the free ones. The energy gap is defined as $\Delta_{F} \equiv \Delta\left(k_{F}\right)$. Eq. (11) can be solved by various techniques, some of which are described in Refs. [7],8]. In Fig. 11 we show the results for $\Delta_{F}$ obtained with the CD-Bonn potential (full line) [17], the Nijmegen I and Nijmegen II potentials (long-dashed line and short-dashed line, respectively) 18]. The results are virtually identical, with the maximum value of the gap varying from $2.98 \mathrm{MeV}$ for the Nijmegen I potential to $3.05 \mathrm{MeV}$ for the Nijmegen II potential. The same insensitivity of the energy gap with respect to the choice of $\mathrm{NN}$ interaction was found in Refs. [4], 8]. We will now discuss how these results can be understood from the properties of the NN interaction in the ${ }^{1} S_{0}$ channel.

A characteristic feature of ${ }^{1} S_{0} \mathrm{NN}$ scattering is the large, negative scattering length, indicating the presence of a nearly bound state at zero scattering energy. Near a bound state, where the NN $T$-matrix has a pole, it can be written in separable form, and this implies that the $\mathrm{NN}$ interaction itself to a good approximation is rankone separable near this pole [19]. Thus at low energies we can write

$$
V\left(k, k^{\prime}\right)=\lambda v(k) v\left(k^{\prime}\right),
$$

where $\lambda$ is a constant. Then it is easily seen from Eq. (11) that the gap function can be written as $\Delta_{F} v(k)$, where $\Delta_{F}$ is the energy gap. Inserting this form of $\Delta(k)$ into Eq. (1) one obtains

$$
1=-\frac{1}{\pi} \int_{0}^{\infty} d k^{\prime} k^{\prime 2} \frac{\lambda v^{2}\left(k^{\prime}\right)}{E\left(k^{\prime}\right)},
$$


which shows that the energy gap $\Delta_{F}$ is determined by the diagonal elements $\lambda v^{2}(k)$ of the NN interaction. The crucial point is that in scattering theory it can be shown that the inverse scattering problem, that is, the determination of a two-particle potential from the knowledge of the phase shifts at all energies, is exactly, and uniquely, solvable for rank-one separable potentials [19,20]. Following the notation of Ref. 19] we have

$$
\lambda v^{2}(k)=-\frac{k^{2}+\kappa_{B}^{2}}{k^{2}} \frac{\sin \delta(k)}{k} e^{-\alpha(k)},
$$

for an attractive potential with a bound state at energy $E=-\kappa_{B}^{2}$. In our case $\kappa_{B}=0$. Here $\delta(k)$ is the ${ }^{1} S_{0}$ phase shift as a function of momentum $k$, while $\alpha(k)$ is given by a principle value integral:

$$
\alpha(k)=\frac{1}{\pi} \mathrm{P} \int_{-\infty}^{+\infty} d k^{\prime} \frac{\delta\left(k^{\prime}\right)}{k^{\prime}-k},
$$

where the phase shifts are extended to negative momenta through $\delta(-k)=-\delta(k)$. Eqs. (4) and (5) can also be rewritten in terms of the Jost function [20] as done in Ref. [21].

From this discussion we see that $\lambda v^{2}(k)$, and therefore also the energy gap $\Delta_{F}$, is completely determined by the ${ }^{1} S_{0}$ phase shifts. However, there are two obvious limitations on the practical validity of this statement. First of all, the separable approximation can only be expected to be good at low energies, near the pole in the $T$-matrix. Secondly, we see from Eq. (5) that knowledge of the phase shifts $\delta(k)$ at all energies is required. This is, of course, impossible, and most phase shift analyses stop at a laboratory energy $E_{\text {lab }}=350 \mathrm{MeV}$. Strictly speaking, the rank-one separable approximation to the ${ }^{1} S_{0}$ interaction breaks down already where the ${ }^{1} S_{0}$ phase shift changes sign from positive to negative at $E_{\text {lab }} \approx 248$ $\mathrm{MeV}$, corresponding to a single-particle momentum of $k \approx 1.73 \mathrm{fm}^{-1}$. However, at low values of $k_{F}$, knowledge of $v(k)$ up to this value of $k$ may actually be enough to determine the value of $\Delta_{F}$, as the integrand in Eq. (5) is strongly peaked around $k_{F}$. We therefore found it worthwhile to try to calculate the energy gap directly from the ${ }^{1} S_{0}$ phase shifts using Eqs. (3)-(5). A possible improvement to the rank-one separable approach for potentials which change sign is discussed by Kwong and Köhler [21].

The input in our calculation is the ${ }^{1} S_{0}$ phase shifts taken from the recent Nijmegen phase shift analysis [22]. We then evaluated $\lambda v^{2}(k)$ from Eqs. (何) and (5), using methods described in Ref. 23] to evaluate the principle value integral in Eq. (5). Finally, we evaluated the energy gap $\Delta_{F}$ for various values of $k_{F}$ by solving Eq. (3). Numerically the integral on the right-hand side of this equation depended very weakly on the momentum structure of $\Delta(k)$, so in our calculations we could take $\Delta(k) \approx \Delta_{F}$ in Eq. (3), and thus it became an algebraic equation for the energy gap $\Delta_{F}$. The resulting energy gap is plotted in Fig. 2 (dashed line) together with the gap obtained with the CD-Bonn potential (full line). As the reader can see, the agreement between the direct calculation from the phase shifts and the CD-Bonn calculation of $\Delta_{F}$ is very good, even at densities as high as $k_{F}=1.4 \mathrm{fm}^{-1}$. The energy gap is to a great extent determined by the available ${ }^{1} S_{0}$ phase shifts. In the same figure we also report the results (dot-dashed line) obtained using the effective range approximation to the phase shifts:

$$
k \cot \delta(k)=-\frac{1}{a_{0}}+\frac{1}{2} r_{0} k^{2},
$$

where $a_{0}=-18.8 \pm 0.3 \mathrm{fm}$ and $r_{0}=2.75 \pm 0.11 \mathrm{fm}$ are the singlet neutron-neutron scattering length and effective range, respectively. In this case an analytic expression can be obtained for $\lambda v^{2}(k)$, as shown in Ref. [20]:

$$
\lambda v^{2}(k)=-\frac{1}{\sqrt{k^{2}+\frac{r_{0}^{2}}{4}\left(k^{2}+\alpha^{2}\right)^{2}}} \sqrt{\frac{k^{2}+\beta_{2}^{2}}{k^{2}-\beta_{1}^{2}}}
$$

with $\alpha^{2}=-2 / a_{0} r_{0}$, and $\beta_{1} \approx-0.0498 \mathrm{fm}^{-1}$, and $\beta_{2} \approx 0.777 \mathrm{fm}^{-1}$ are the two roots of the quadratic equation

$$
\beta^{2}-\frac{2}{r_{0}} \beta-\alpha^{2}=0
$$

The phase shifts using this approximation are positive at all energies, and this is reflected in Eq. (]) where $\lambda v^{2}(k)$ is attractive for all $k$. From Fig. 1 we see that below $k_{F}=0.5 \mathrm{fm}^{-1}$ the energy gap can with reasonable accuracy be calculated with the interaction obtained directly from the effective range approximation. One can therefore say that at densities below $k_{F}=0.5 \mathrm{fm}^{-1}$, and at the crudest level of sophistication in many-body theory, the superfluid properties of neutron matter are determined by just two parameters, namely the free-space scattering length and effective range. At such densities, more complicated many-body terms are also less important. Also interesting is the fact that the phase shifts predict the position of the first zero of $\Delta(k)$ in momentum space, since we see from Eq. (四) that $\Delta(k)=\Delta_{F} v(k)=0$ first for $\delta(k)=0$, which occurs at $E_{\text {lab }} \approx 248 \mathrm{MeV}$ (pp scattering) corresponding to $k \approx 1.73 \mathrm{fm}^{-1}$. This is in good agreement with the results of Khodel et al. [8]. In Ref. [8] it is also shown that this first zero of the gap function determines the Fermi momentum at which $\Delta_{F}=0$. Our results therefore indicate that this Fermi momentum is in fact given by the energy at which the ${ }^{1} S_{0}$ phase shifts become negative.

The calculation of the ${ }^{1} S_{0}$ gap in symmetric nuclear matter is closely related to the one for neutron matter. In fact, with charge-independent forces, like the older Bonn potentials, and free single-particle energies one would, of course, obtain exactly the same results. However, the new potentials on the market are charge-dependent, in order to achieve high quality fits to both np and pp scattering data, and therefore we must solve three coupled 
gap equations for neutron-neutron (nn), proton-proton (pp), and neutron-proton (np) pairing 24]:

$$
\Delta_{i}(k)=-\frac{1}{\pi} \int_{0}^{\infty} d k^{\prime} k^{\prime 2} V_{i}\left(k, k^{\prime}\right) \frac{\Delta_{i}\left(k^{\prime}\right)}{E\left(k^{\prime}\right)},
$$

where $i=\mathrm{nn}, \mathrm{pp}$ and np, and the quasiparticle energy is still given by $E(k)=\sqrt{\left(\epsilon(k)-\epsilon\left(k_{F}\right)\right)^{2}+\Delta(k)^{2}}$, but the energy gap is now given by

$$
\Delta(k)^{2}=\Delta_{\mathrm{nn}}(k)^{2}+\Delta_{\mathrm{pp}}(k)^{2}+\Delta_{\mathrm{np}}(k)^{2} .
$$

Thus the equations are coupled through their common energy denominator. The ${ }^{1} S_{0} \mathrm{pp}$ and nn interactions are very nearly identical, so the set of equations above can be reduced to two: one for the nn (or pp) gap and one for the np gap. Solving these equations, both with the CD-Bonn potential and with the phase shift approximations we get the results shown in Fig. 3. For comparison we have in the same figure plotted the results for pure neutron matter with the CD-Bonn potential (dashed line). From the figure it is clear that the phase shift approximation works well also in this case, and that the gap in symmetric matter is not very different from the gap in neutron matter. As could be expected, the results are very close to those obtained earlier with charge-independent interactions [7].

In summary, we have shown that in infinite neutron and nuclear matter, owing to the near rank-one separability of the NN interaction in the ${ }^{1} S_{0}$ partial wave, we are able to compute the ${ }^{1} S_{0}$ pairing gap directly from the NN phase shifts. This explains also why all NN potentials which fit the scattering data result in almost identical ${ }^{1} S_{0}$ pairing gaps. This is the main result of this work. However, it should be mentioned that this result is not likely to survive in a more refined calculation, for instance if one includes density and spin-density fluctuations in the effective pairing interaction like in e.g., Refs. [13,14. Other partial waves will then be involved, and the simple arguments employed here will no longer apply. Our reasoning here applies also only to a partial wave where the $T$-matrix (almost) has a pole, and we have neglected the fact that the phase shifts become negative at higher energies. As a curiosity, we have found that at Fermi momenta below $0.5 \mathrm{fm}^{-1}$ the pairing gap is even determined by two parameters only, the effective range and the scattering length. Also, we have pointed out that since the new NN interactions are charge dependent, one has to consider three coupled gap equations for ${ }^{1} S_{0}$ pairing in nuclear matter. The final result though is very nearly the same has what one obtains with charge-independent interactions.

We are much indebted to J. W. Clark, and E. Osnes for many valuable comments and discussions.
[1] C. J. Pethick and D. G. Ravenhall, Annu. Rev. Nucl. Part. Sci. 45, 429 (1995).

[2] A. C. Müller and B. M. Sherril, Annu. Rev. Nucl. Part. Sci. 43, 529 (1993).

[3] K. Riisager, Rev. Mod. Phys. 66, 1105 (1994).

[4] M. Baldo, J. Cugnon, A. Lejeune, and U. Lombardo, Nucl. Phys. A 515, 409 (1990).

[5] J. M. C. Chen, J. W. Clark, R. D. Davé, and V. V. Khodel, Nucl. Phys. A 555, 59 (1993).

[6] T. Takatsuka and R. Tamagaki, Prog. Theor. Phys. Suppl. 112, 27 (1993).

[7] Ø. Elgarøy, L. Engvik, M. Hjorth-Jensen, and E. Osnes, Nucl. Phys. A 604, 466 (1996).

[8] V. A. Khodel, V. V. Khodel, and J. W. Clark, Nucl. Phys. A 598, 390 (1996).

[9] H. Kucharek and P. Ring, Z. Phys. 339, 23 (1990).

[10] F. B. Guimarães, B. V. Carlson, and T. Frederico, Phys. Rev. C 54, 2385 (1996).

[11] F. Matera, G. Fabbri, and A. Dellafiore, Phys. Rev. C 56, 228 (1997).

[12] J. W. Clark, C. G. Källman, C.H. Yang, and D. A. Chakkalakal, Phys. Lett. B 61, 331 (1976).

[13] J. Wambach, T. L. Ainsworth, and D. Pines, Nucl. Phys. A 555, 128 (1993).

[14] H.J. Schulze, J. Cugnon, A. Lejeune, M. Baldo, and U. Lombardo, Phys. Lett. B 375, 1 (1996).

[15] V. J. Emery and A. M. Sessler, Phys. Rev. 119, 248 (1960) and references therein.

[16] M. Hoffberg, A. E. Glassgold, R. W. Richardson, and M. Ruderman, Phys. Rev. Lett. 24, 775 (1970).

[17] R. Machleidt, F. Sammarruca, and Y. Song, Phys. Rev. C 53, 1483 (1996).

[18] V. G. J. Stoks, R. A. M. Klomp, C. P. F. Terheggen, and J. J. de Swart, Phys. Rev. C 49, 2950 (1994); NN-Online facility, (URL: http://NN-OnLine.sci.kun.nl).

[19] G. E. Brown and A. D. Jackson, The Nucleon-Nucleon Interaction (North-Holland, Amsterdam, 1976).

[20] K. Chadan and P. C. Sabatier, Inverse Problems in Quantum Scattering Theory, 2nd ed. (Springer, New York, 1992).

[21] N. H. Kwong and H. S. Köhler, Phys. Rev. C 55, 1650 (1997).

[22] V. G. J. Stoks, R. A. M. Klomp, M. G. M. Rentmeester, and J. J. de Swart, Phys. Rev. C 48, 792 (1993).

[23] K. T. R. Davies, G. D. White, and R. W. Davies, Nucl. Phys. A 524, 743 (1991).

[24] A. L. Goodman, Adv. Nucl. Phys. 11, 263 (1979). 


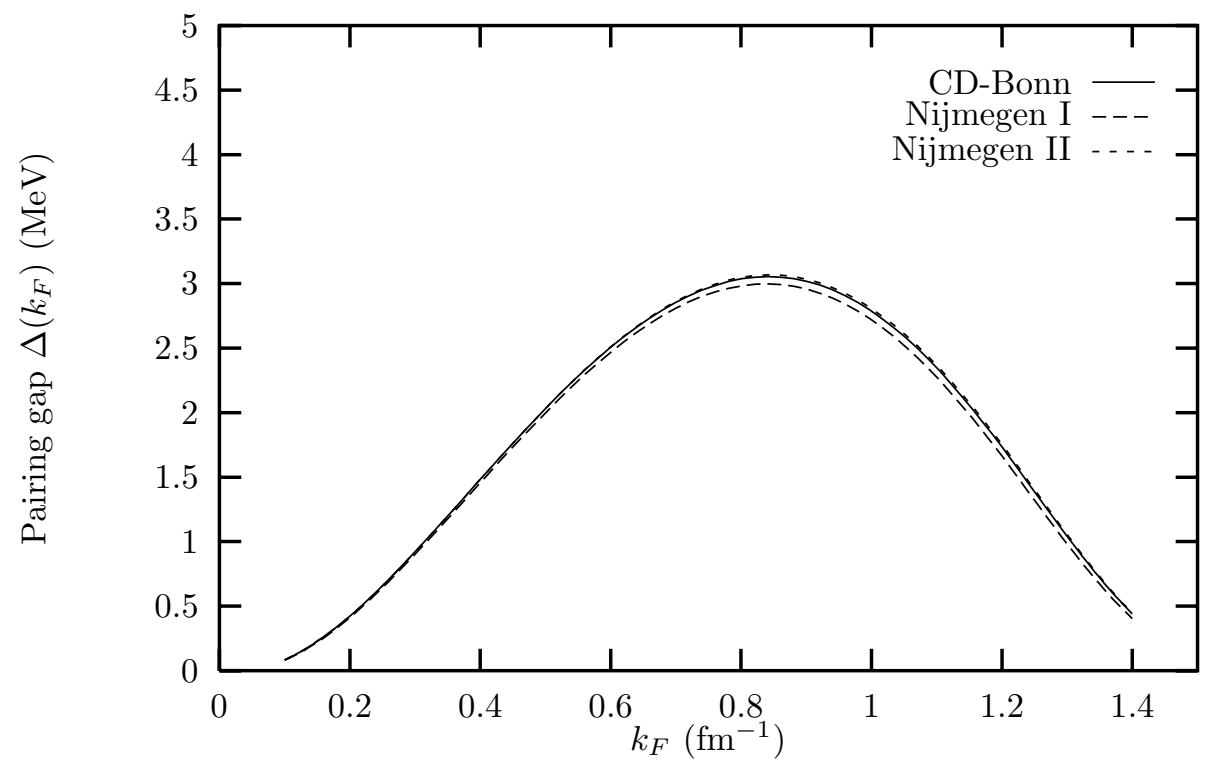

FIG. 1. ${ }^{1} S_{0}$ energy gap in neutron matter with the CD-Bonn, Nijmegen I and Nijmegen II potentials.

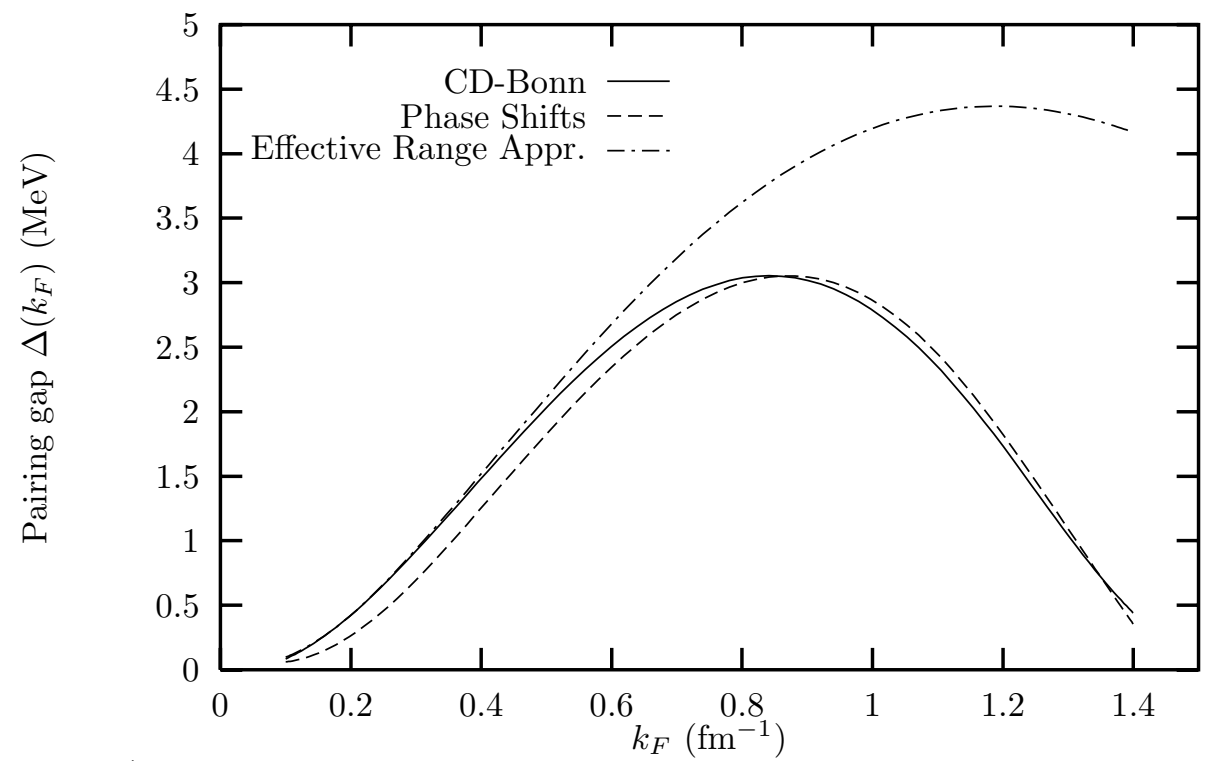

FIG. 2. ${ }^{1} S_{0}$ energy gap in neutron matter calculated with the CD-Bonn potential compared with the direct calculation from ${ }^{1} S_{0}$ phase shifts. 


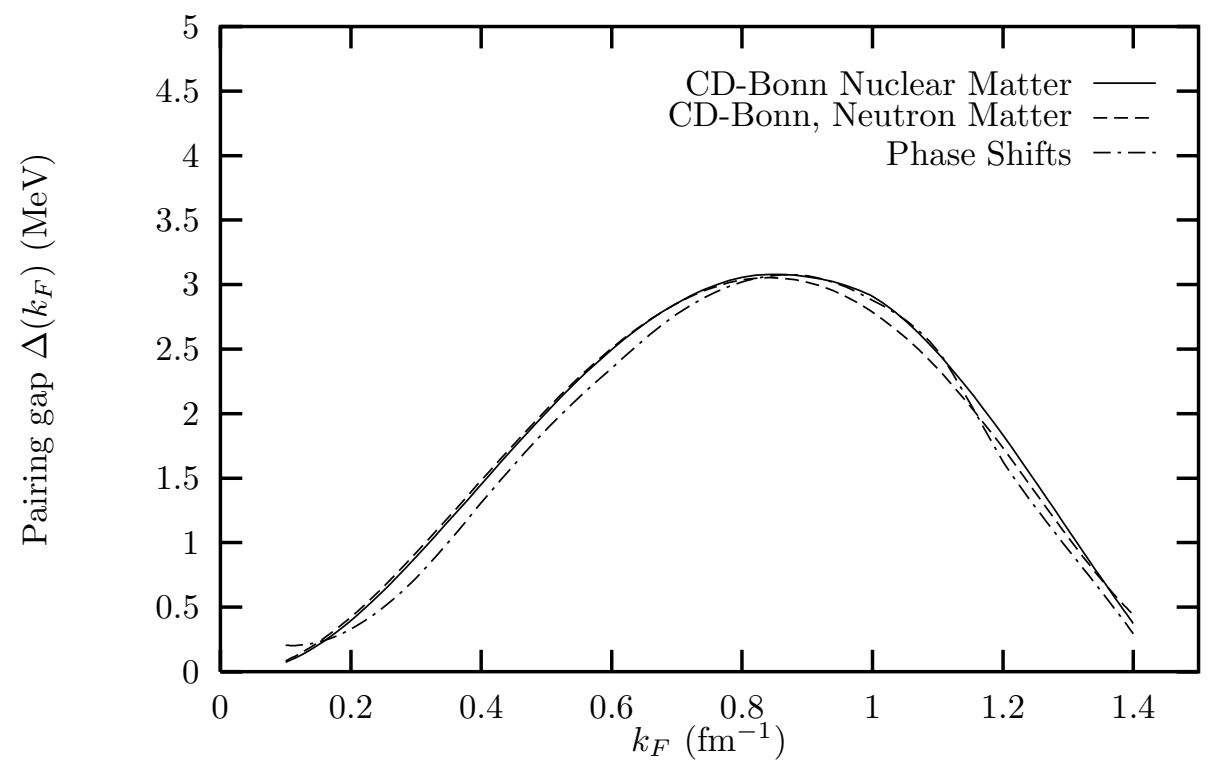

FIG. 3. ${ }^{1} S_{0}$ energy gap in nuclear matter calculated with the CD-Bonn potential compared with the direct calculation from the ${ }^{1} S_{0}$ np and pp phase shifts. Also shown are the results for neutron matter with the CD-Bonn potential. 